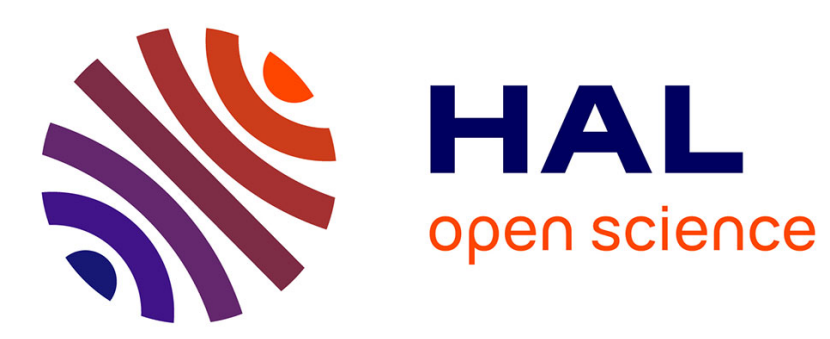

\title{
Square roots of minor closed graph classes
}

Nestor Nestoridis, Dimitrios M. Thilikos

\section{To cite this version:}

Nestor Nestoridis, Dimitrios M. Thilikos. Square roots of minor closed graph classes. Discrete Applied Mathematics, 2014, 168, pp.34 - 39. 10.1016/j.dam.2013.05.026 . hal-01083980v2

\section{HAL Id: hal-01083980 \\ https://hal.science/hal-01083980v2}

Submitted on 6 Nov 2015

HAL is a multi-disciplinary open access archive for the deposit and dissemination of scientific research documents, whether they are published or not. The documents may come from teaching and research institutions in France or abroad, or from public or private research centers.
L'archive ouverte pluridisciplinaire HAL, est destinée au dépôt et à la diffusion de documents scientifiques de niveau recherche, publiés ou non, émanant des établissements d'enseignement et de recherche français ou étrangers, des laboratoires publics ou privés. 


\title{
Square Roots of Minor Closed Graph Classes
}

\author{
Nestor V. Nestoridis ${ }^{\mathrm{a}}$, Dimitrios M. Thilikos ${ }^{\mathrm{a}}$ \\ ${ }^{a}$ Department of Mathematics, National and Kapodistrian University of Athens, \\ Panepistimioupolis, GR-15784 Athens, Greece
}

\begin{abstract}
Let $\mathcal{G}$ be a graph class. The square root of $\mathcal{G}$ contains all graphs whose square belong in $\mathcal{G}$. We prove that if $\mathcal{G}$ is non-trivial and minor closed, then all graphs in its square root have carving-width bounded by some constant depending only on $\mathcal{G}$. As a consequence, every square root of such a graph class has a linear time recognition algorithm.
\end{abstract}

Keywords: square roots of graphs, branch-width, carving-width, graph minors.

\section{Introduction}

Let $\mathcal{G}$ be a graph class. The square root of $\mathcal{G}$ is defined as the graph class

$$
\sqrt{\mathcal{G}}=\left\{G \mid G^{2} \in \mathcal{G}\right\}
$$

where the square $G^{2}$ of a graph $G$ is the graph obtained from $G$ after adding edges between all pairs of vertices that share a common neighbor.

In [3], Harary, Karp, and Tutte provided a complete characterization of the graphs in $\sqrt{\mathcal{P}}$ where $\mathcal{P}$ is the class of all planar graphs. Notice that planar graphs are minor closed, i.e. a minor of every graph in $\mathcal{P}$ also belongs in $\mathcal{P}$.

According to the characterization of [3], all graphs in $\sqrt{\mathcal{P}}$ are outerplanar and have bounded degree. This implies that graphs in $\sqrt{\mathcal{P}}$ have a very specific "tree-like" structure and it is a natural question whether this is the case for more general graph classes. In this paper we extend this result, in the sense that the same tree-like property holds for every minor closed graph class that is non-trivial (i.e. that does not contain all graphs). In

Email addresses: nestornestoridis@yahoo.gr (Nestor V. Nestoridis), sedthilk@math.uoa.gr (Dimitrios M. Thilikos) 
fact, we prove (in Section 3) that, in this case, the correct "tree-likeness" property if given by the parameter of carving-width, introduced by Seymour and Thomas in [10]. As a consequence, we prove in Section 4 that the square root of any non-trivial minor closed graph class has a linear time recognition algorithm. This extends the algorithmic results of [5] where a linear time algorithm was given for recognizing the square roots of planar graphs.

\section{Definitions}

We next give some definitions that are necessary in order to formally define carving width. This will permit us to give the formal statement of our combinatorial result.

Boundaries in graphs and hypergraphs. In this paper we deal with graphs and hypergraphs. For a (hyper)graph $G$ we denote by $V(G)$ its vertex set and by $E(G)$ the set of its (hyper)edges. If $S \subseteq V(G)$ (resp. $F \subseteq E(G)$ ) we denote $\bar{S}=V(G) \backslash S($ resp. $\bar{F}=E(G) \backslash F)$.

Given a vertex set $S \subseteq V(G)$, we define by $E_{G}(S)$ as the set of hyperedges containing vertices in $S$. For simplicity we also denote $E_{G}(v)=E_{G}(\{v\})$. We also define $\Delta(G)=\max \left\{\left|E_{G}(v)\right| \mid v \in V(G)\right\}$. Given a set $S \subseteq V(G)$, we define

$$
\partial_{G}(S)=E_{G}(S) \cap E_{G}(\bar{S}) .
$$

Notice that $\partial_{G}$ is a symmetric function, i.e. for every $S \subseteq V(G)$, ti holds that $\partial_{G}(S)=\partial_{G}(\bar{S})$. Also given a set $F \subseteq E(G)$, we set

$$
\partial_{G}^{*}(F)=\left(\bigcup_{f \in F} f\right) \cap\left(\bigcup_{f \in \bar{F}} f\right)
$$

Given a hypergraph $G$ we define its dual as the hypergraph

$$
G^{*}=\left(E(G),\left\{E_{G}(v) \mid v \in V(G)\right\}\right) .
$$

Notice that the hypergraphs $G$ and $G^{*}$ have the same incidence graph with the roles of their two parts reversed. Given a set $S \subseteq V(G)$ (resp. $F \subseteq$ $E(G))$ we denote by $S^{*}$ (resp. $F^{*}$ ) their dual hyperedges (resp. vertices) in $G^{*}$.

Using duality, we also define $\Delta^{*}(G)=\Delta\left(G^{*}\right)$. Clearly, for a simple graph $G, \Delta^{*}(G)=2$. Moreover, the above definitions imply that for every $F \subseteq E(G),\left(\partial_{G}^{*}(F)\right)^{*}=\partial_{G^{*}}\left(F^{*}\right)$.

A graph $H$ is a minor of a graph $G$, and we write $H \leq G$, if $H$ can be obtained by some subgraph of $G$ after contracting edges (the contracting an 
edge $e=\{x, y\}$ is the operation that introduces removes $x$ and $y$ from $G$ and introduces a new vertex $v_{e}$ that is made adjacent with all the neighbors of $x$ and $y$ in $G$, except from $x$ and $y$ ). A graph class $\mathcal{G}$ is minor closed if every minor of a graph in $\mathcal{G}$ is also a graph in $\mathcal{G}$.

Carving-width. Given a tree $T$ we denote the set of its leaves by $L(T)$ and we call it ternary if all vertices in $V(T) \backslash L(T)$ have degree 3. A carving decomposition of a hypergraph $G$ is a pair $(T, \rho)$, where $T$ is a ternary tree and $\rho$ is a bijection from $V(G)$ to $L(T)$. The bridge function $\beta: E(T) \rightarrow 2^{E(G)}$ of a carving decomposition maps every edge $e$ of $T$ to the set $\partial_{G}\left(\rho^{-1}\left(L\left(T^{\prime}\right)\right)\right)$ where $T^{\prime}$ is one of the two connected components of $T \backslash e$. The width of $(T, \rho)$ is equal to $\max _{e \in E(T)}|\beta(e)|$ and the carving-width of $G, \mathbf{c w}(G)$, is the minimum width over all carving decompositions of $G$. The following observation is a direct consequence of the definitions.

Observation 1. For every hypergraph $G$, it holds that $\Delta(G) \leq \mathbf{c w}(G)$.

The main combinatorial result of this paper is the following.

Theorem 1. For every non-trivial minor closed graph class $\mathcal{G}$ there is a constant $c_{\mathcal{G}}$ such that all graphs is $\sqrt{\mathcal{G}}$ have carving-width at most $c_{\mathcal{G}}$.

The proof of Theorem 1 uses the parameter of branch-width defined in [7].

Branch-width. A branch decomposition of a graph $G$ is a pair $(T, \tau)$, where $T$ is a ternary tree and $\tau$ is a bijection from $E(G)$ to $L(T)$. The boundary function $\omega: E(T) \rightarrow 2^{V(G)}$ of a branch decomposition maps every edge $e$ of $T$ to the set $\partial_{G}^{*}\left(\rho^{-1}\left(L\left(T^{\prime}\right)\right)\right)$ where $T^{\prime}$ is one of the two connected components of $T-\{e\}$. The width of $(T, \tau)$ is equal to $\max _{e \in E(T)}|\omega(e)|$ and the branchwidth of $G$, bw $(G)$, is the minimum width over all branch decompositions of $G$.

The following observation is a direct consequence of the duality between the functions $\partial_{G}$ and $\partial_{G}^{*}$.

Observation 2. For every hypergraph $G$ it holds that $\mathbf{b w}(G)=\mathbf{c w}\left(G^{*}\right)$.

\section{Walls and squares}

Walls. A wall of height $k, k \geq 1$, is obtained from a $((k+1) \times(2 k+2))$-grid with vertices $(x, y), x \in\{0, \ldots, 2 k+1\}, y \in\{0, \ldots, k\}$, after removing the "vertical" edges $\{(x, y),(x, y+1)\}$ for odd $x+y$. We denote such a wall by 
$W_{k}$. A subdivided wall of height $k$ is obtained by the wall $W_{k}$ with some edges of $W_{k}$ replaced by paths without common internal vertices. If, in such a subdivided wall, all edges have been subdivided at least once, then we say that it is properly subdivided.

The following result follows from the results in [6] and [7].

Proposition 1 ([6] and [7])). There is a function $g: \mathbb{N} \rightarrow \mathbb{N}$ such that every graph $G$ with branchwidth at least $g(k)$ contains the $(k \times k)$-grid as a minor.

A direct consequence of Proposition 1 is the following.

Lemma 1. There exists a function $f$ such that every graph $G$ with branchwidth at least $f(k)$ contains a properly subdivided wall of height $k$ as a subgraph.

Proof. Let $W_{k}^{\prime}$ be the graph taken from a $k$-wall if we subdivide each edge once. Notice that $W_{k}^{\prime}$ is a subgraph of the $((4 k+3) \times(4 k+3))$-grid. The lemma follows from Proposition 1 and the fact that minor relation and topological minor relation are identical when the host graph has maximum degree 3 (notice that $\Delta\left(W_{k}^{\prime}\right) \leq 3$ ).

Let $H$ be a $((k+1) \times(2 k+2))$-grid where $k$ is a positive odd integer. Let also $W_{k}$ a spanning subgraph that is a $k$-wall as explained above. Let also $G$ be a subdivision of $W_{k}$ where the non-subdivision vertices are denote as coordinates of $H$ (we call these vertices original). In particular, we denote the vertex of $H$ with coordinates $i, j$ by $a^{i, j}$ (see Figure1). For $i \in\{0, \ldots, k-$ $1\}$ and $j \in\{0, \ldots, 2 k+1\}$ where $i+j$ is even, we denote by $P_{i, j}^{(v)}$ the path that has replaced the edge $\left\{a^{i, j}, a^{i+1, j}\right\}$ in $W_{k}$ and we call these paths vertical. For $i \in\{0, \ldots, k\}$ and $j \in\{0, \ldots, 2 k\}$, we denote by $P_{i, j}^{(h)}$ the path that has replaced the edge $\left\{a^{i, j}, a^{i, j+1}\right\}$ and we call these paths horizontal We denote by $L_{i}, i \in\{0, \ldots, k\}$ the path $P_{i, 0}^{h} \oplus \cdots \oplus P_{i, 2 k}^{h}$, we direct it from $a^{i, 0}$ to $a^{i, 2 k}$ and we call it $i$-th line of $G$. We also define, for $l \in\{0, \ldots, k\}$, the $l$-th meander of $G$ as the path

$$
\begin{aligned}
M_{l}= & P_{0,2 l}^{(v)} \oplus P_{1,2 l}^{(h)} \oplus P_{1,2 l+1}^{(v)} \oplus P_{2,2 l}^{(h)} \oplus P_{2,2 l}^{(v)} \oplus \ldots \\
& \oplus P_{k-2,2 l+1}^{(v)} \oplus P_{k-1,2 l}^{(h)} \oplus P_{k-1,2 l}^{(v)} .
\end{aligned}
$$

Finally, for every original vertex $a^{i, j}$ where $0 \leq j \leq 2 k(1 \leq j \leq 2 k+1)$, we define $\operatorname{right}_{G}\left(a^{i, j}\right)\left(\operatorname{left}_{G}\left(a^{i, j}\right)\right)$ as the vertex of the directed path $L_{i}$ that appears right after (before) $a^{i, j}$. 


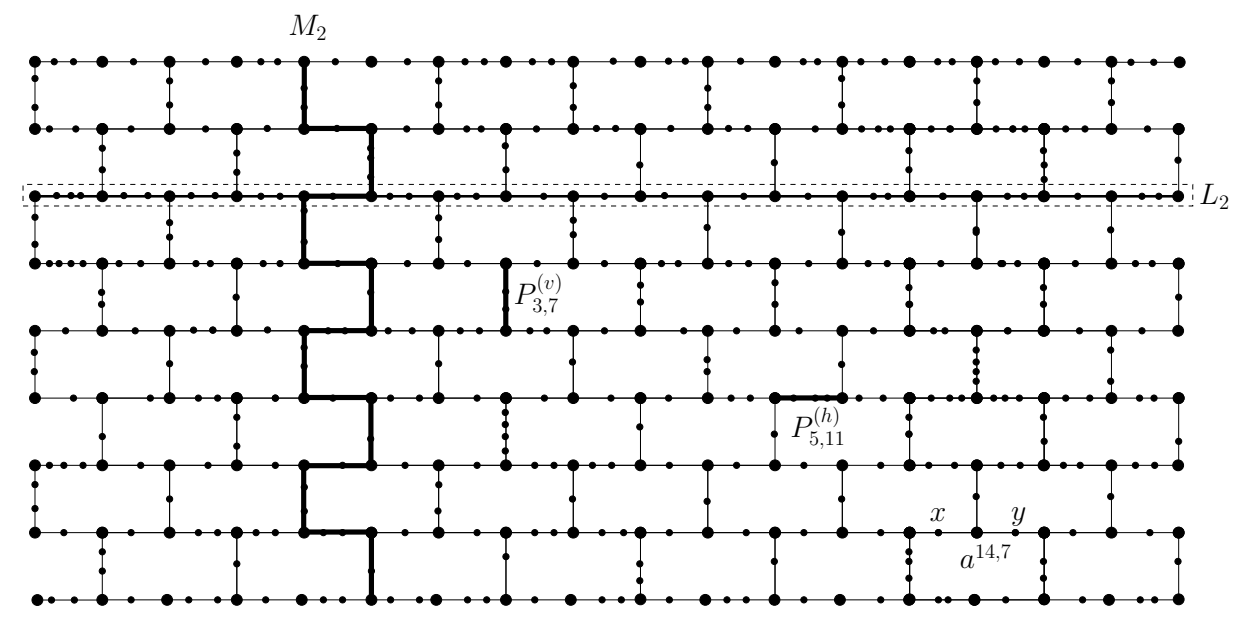

Figure 1: A properly subdivided wall $G$ of height 8 . The big (resp. small) vertices are the original (subdivision) vertices. The paths $P_{3,7}^{(v)}, P_{5,11}^{(h)}, L_{2}$ and the 2-meander $M_{2}$ are depicted in $G$. Notice that $x=\operatorname{left}_{G}\left(a^{14,7}\right)$ and $y=\operatorname{right}_{G}\left(a^{14,7}\right)$.

We denote by $K_{r}$ the complete graph on $r$ vertices. Also we use the notation $K_{r, q}$ for the complete bipartite graph with parts of size $r$ and $q$.

Lemma 2. Let $k$ be a positive odd integer. If $G$ is a properly subdivided wall of height $k$, then $K_{k+2} \leq G^{2}$.

Proof. For each $i \in\{0, \ldots, k\}$ we define the path $P_{i}$ of $G^{2}$ as follows: If $L_{i}=$ $\left(x_{0}, x_{1}, \ldots, x_{m}\right)$ is the $i$-th horizontal path of $G$, then $\bar{L}_{i}=\left(x_{0}, x_{2}, x_{4}, \ldots, x_{m^{\prime}}\right)$ where $m^{\prime}=m$ or $m^{\prime}=m-1$ depending on whether $m$ is even or not. We set $S=\bigcup_{i \in\{0, \ldots, k\}} V\left(\bar{L}_{i}\right)$.

Our next step is to define the paths $Q_{j}$ of $G^{2}, l \in\{0, \ldots, k\}$. Let $M_{l}$ be the $l$-th meander of $G$. Following the definition of a meander, we denote

- $P_{i, 2 l}^{(v)}=\left(y_{1}^{i, 2 l}, \ldots, y_{w_{i, 2 l}}^{i, 2 l}\right)$, for even $i \in\{0, \ldots, k-1\}$ (assuming that $y_{1}^{i, 2 l}=a^{i, 2 l}$ and $\left.y_{w_{i, 2 l}}^{i, 2 l}=a^{i+1,2 l}\right)$,

- $P_{i, 2 l+1}^{(v)}=\left(y_{1}^{i, 2 l+1}, \ldots, y_{w_{i, 2 l+1}}^{i, 2 l+1}\right)$, for odd $i \in\{0, \ldots, k-2\}$ (assuming that $y_{1}^{i, 2 l+1}=a^{i, 2 l+1}$ and $\left.y_{w_{i, 2 l+1}}^{i, 2 l+1}=a^{i+1,2 l+1}\right)$,

- $P_{i, 2 l}^{(h)}=\left(z_{1}^{i, 2 l}, \ldots, z_{m_{i, 2 l}}^{i, 2 l}\right), i \in\{1, \ldots, k-1\}$ (assuming that $z_{1}^{i, 2 l}=a^{i, 2 l}$ and $\left.z_{m_{i, 2 l}}^{i, 2 l}=a^{i, 2 l+1}\right)$. 
For each even $i \in\{0, \ldots, k-1\}$, we set $\bar{P}_{i, 2 l}^{(v)}=\left(\bar{y}_{1}^{i, 2 l}, y_{2}^{i, 2 l}, \ldots, y_{w_{i, 2 l}-1}^{i, 2 l}, \bar{y}_{w_{i, 2 l}}^{i, 2 l}\right)$ where

$$
\begin{gathered}
\bar{y}_{1}^{i, 2 l}= \begin{cases}y_{1}^{i, 2 l} & \text { if } y_{1}^{i, 2 l} \notin S \\
\operatorname{right}_{G}\left(y_{1}^{i, 2 l}\right) & \text { if } y_{1}^{i, 2 l} \in S\end{cases} \\
\bar{y}_{w_{i, 2 l}, 2 l}= \begin{cases}y_{w_{i, 2 l}}^{i, 2 l} & \text { if } y_{w_{i, 2 l}}^{i, 2 l} \notin S \\
\operatorname{right}_{G}\left(y_{w_{i, 2 l}}^{i, 2 l}\right) & \text { if } y_{1}^{i, 2 l} \in S .\end{cases}
\end{gathered}
$$

and for each odd $i \in\{0, \ldots, k-1\}$, we set $\bar{P}_{i, 2 l+1}^{(v)}=\left(\bar{y}_{1}^{i, 2 l+1}, y_{2}^{i, 2 l+1}, \ldots\right.$ $\left., y_{w_{x i, 2 l}-1}^{i, 2 l+1}, \bar{y}_{w_{i, 2 l+1}}^{i, 2 l+1}\right)$ where

$$
\begin{gathered}
\bar{y}_{1}^{i, 2 l+1}= \begin{cases}y_{1}^{i, 2 l+1} & \text { if } y_{1}^{i, 2 l+1} \notin S \\
\operatorname{left}_{G}\left(y_{1}^{i, 2 l+1}\right) & \text { if } y_{1}^{i, 2 l+1} \in S\end{cases} \\
\bar{y}_{w_{i, 2 l+1}}^{i, 2 l+1}= \begin{cases}y_{w_{i, 2 l+1}}^{i, 2 l+1} & \text { if } y_{w_{i, 2 l+1}}^{i, 2 l+1} \notin S \\
\operatorname{left}_{G}\left(y_{w_{i, 2 l+1}}^{i, 2 l+1}\right) & \text { if } y_{w_{i, 2 l+1}}^{i, 2 l+1} \in S .\end{cases}
\end{gathered}
$$

Observe that for each $i \in\{1, \ldots, k-1\}$ and $l \in\{0, \ldots, k\}$ the vertices in $P_{i, 2 l}^{(h)} \backslash S$ induce a path in $G^{2}$ which we denote by $\bar{P}_{i, 2 l}^{(h)}$.

Notice, that for every odd $i \in\{1, \ldots, k-2\}$ it holds that

$$
\begin{aligned}
V\left(\bar{P}_{i-1,2 l}^{(v)}\right) \cap V\left(\bar{P}_{i, 2 l}^{(h)}\right) & =\left\{\bar{y}_{w_{i-1,2 l}}^{i-1,2 l}\right\} \\
V\left(\bar{P}_{i, 2 l}^{(h)}\right) \cap V\left(P_{i, 2 l+1}^{(v)}\right) & =\left\{\bar{y}_{1}^{i, 2 l+1}\right\} .
\end{aligned}
$$

Also, for every even $i \in\{2, \ldots, k-1\}$ is also holds that

$$
\begin{aligned}
V\left(\bar{P}_{i-1,2 l+1}^{(v)}\right) \cap V\left(\bar{P}_{i, 2 l}^{(h)}\right) & =\left\{\bar{y}_{w_{i-1,2 l+1}}^{i-1,2 l+1}\right\} \\
V\left(\bar{P}_{i, 2 l}^{(h)}\right) \cap V\left(\bar{P}_{i, 2 l}^{(v)}\right) & =\left\{\bar{y}_{1}^{i, 2 l}\right\} .
\end{aligned}
$$

From (1)-(4) we obtain that

$$
\bar{M}_{j}=\bar{P}_{0,2 l}^{(v)} \oplus \bar{P}_{1,2 l}^{(h)} \oplus \bar{P}_{1,2 l+1}^{(v)} \oplus \bar{P}_{2,2 l}^{(h)} \oplus \bar{P}_{2,2 l}^{(v)} \oplus \ldots \oplus \bar{P}_{k-2,2 l+1}^{(v)} \oplus \bar{P}_{k-1,2 l}^{(h)} \oplus \bar{P}_{k-1,2 l}^{(v)}
$$

is a path in $G^{2}$ for each $l \in\{0, \ldots, k\}$.

As paths in $\overline{\mathcal{M}}=\left\{\bar{M}_{l} \mid l \in\{0, \ldots, k\}\right\}$ avoid all vertices of $S$, we have that for each $i, l \in\{0, \ldots, k\} V\left(\bar{L}_{i}\right) \cap V\left(\bar{M}_{l}\right)=\emptyset$. Moreover, by construction, any two paths in $\mathcal{M}$ are vertex disjoint. Recall also that the same holds for the paths in $\overline{\mathcal{L}}=\left\{\bar{L}_{i} \mid i \in\{0, \ldots, k-1\}\right\}$. 
We claim that for each $i, l \in\{0, \ldots, k\}$ there is an edge $e_{i, l}$ in $G^{2}$ with one endpoint in $\bar{L}_{i}$ and the other in $\bar{M}_{l}$. Indeed it is easy to see that one can take

$$
e_{i, l}=\left\{\begin{array}{lll}
\left\{a^{i, 2 l}, \operatorname{left}\left(a^{i, 2 l}\right)\right\} & \text { if }(i, l) \in\{(0, k),(k, k)\} \\
& \left\{a^{i, 2 l}, \operatorname{right}\left(a^{i, 2 l}\right)\right\} & \text { otherwise }
\end{array}\right.
$$

Now we are ready to prove that $G^{2}$ contains $K_{k+2}$ as a minor. For this, we first remove from $G^{2}$ all edges that are neither edges of paths in $\overline{\mathcal{M}} \cup \overline{\mathcal{L}}$ nor edges in $\mathcal{E}=\left\{e_{i, l} \mid(i, l) \in\{0, \ldots, k\}^{2}\right\}$. Then we contract all edges of the remaining graph except from those in $\mathcal{E}$ and we end up to a graph isomorhpic to $K_{k+1, k+1}$ that, in turn, can be further contracted to $K_{k+2}$.

Extended carving-decompositions. Let us relax the definition of a carving decomposition in the case of graphs in the following way: let $G$ be a graph, $T$ be a ternary tree rooted on some, say $r$, of its leaves, and a surjection $\sigma: L(T) \backslash\{r\} \rightarrow V(G)$. As $T$ is rooted each edge of $T$ can be seen as a directed edge pointing towards the root. Let $f=(x, y)$ be such an edge and let $T_{x}$ and $T_{y}$ be the two connected components of $T \backslash f$ such that $r \in V\left(T_{y}\right)$. Let also $V_{x}=\sigma\left(L\left(T_{x}\right)\right)$ and $V_{y}=\sigma\left(L\left(T_{y}\right)\right)$. We define the bridge set of $f$ as $\beta(f)=\left\{\{a, b\} \in E(G) \mid a \in V_{x}\right.$ and $\left.b \in V_{y} \backslash V_{x}\right\}$. We call each such pair $(T, \sigma)$ an extended carving decomposition of $G$ and we define its width as the maximum $|\beta(f)|$ over all $f \in E(T)$.

Lemma 3. If $G$ has an extended carving decomposition of width at most $k$, then it also has a carving decomposition of width at most $k$.

Proof. Let $(T, \sigma)$ be such an extended carving decomposition. We apply on $(T, \sigma)$ the following transformation until this is not possible any more: if for some $v$, in $\sigma^{-1}(v)$ contains at least two vertices $a$ and $b$ then replace $\sigma$ by $\sigma \backslash\{(a, v)\}$ and replace $T$ by the tree that is obtained if we remove $a$ and then we dissolve the resulting vertex of degree 2 . It is easy to see that each new pair $(T, \sigma)$ has width at most $k$. Also when the above procedure finishes, $\sigma^{-1}$ is a bijection from $V(G)$ to $L(T) \backslash\{r\}$. If now we remove $r$ from $T$ and dissolve the resulting vertex of degree 2, we have a branch decomposition $\left(T^{\prime}, \sigma^{-1}\right)$ of $G$ with width at most $k$.

Lemma 4. For every graph $G, \mathbf{c w}(G) \leq \Delta(G) \cdot \mathbf{b w}(G)$. 
Proof. We set $\delta=\Delta(G)$. Let $(T, \tau)$ be a branch decomposition of $G$ with width at most $k$. We now define an extended carving decomposition $\left(T^{\prime}, \sigma\right)$ of $G$ as follows: Take $T$ and for each $v \in L(T)$ we define two new vertices $v_{1}$ and $v_{2}$ and we make them adjacent to $v$. For each such $v$ where $\tau^{-1}(v)=\{z, w\}$, we set $\sigma\left(v_{1}\right)=z$ and $\sigma\left(v_{2}\right)=w$. In the resulting tree we pick arbitrarily an edge, we subdivide it and we make the subdivision vertex adjacent with new vertex $r$. In the resulting graph $T^{\prime}, r$ will be the root. Let $f=(x, y)$ be an edge of $T^{\prime}$. We set $X=\sigma\left(L\left(T_{x}\right)\right) \backslash \sigma\left(L\left(T_{y}\right)\right)$, $Y=\sigma\left(L\left(T_{y}\right)\right) \backslash \sigma\left(L\left(T_{x}\right)\right)$ and $S=\sigma\left(L\left(T_{x}\right)\right) \cap \sigma\left(L\left(T_{y}\right)\right)$. Notice that $\omega(f)$, as defined by the branch decomposition $(T, \mu)$, is exactly the set $S$, therefore $S$ is a separator of $G$ and $|S| \leq k$. We conclude that each edge in $\omega(f)$ has one endpoint in $S$ and the other in $Y$. As each vertex in $S$ has degree $\leq \Delta(G)$, we obtain that $\omega(f) \leq k \cdot d$. Therefore, $\left(T^{\prime}, \sigma\right)$ is an extended branch decomposition of $G$ with width $\leq k \cdot d$. The lemma now follows from Lemma 3 ,

We are now ready to prove the main result of this paper.

Proof of Theorem 1. As $\mathcal{G}$ is non-trivial class we choose a graph $H \notin \mathcal{G}$ and we assume that $H$ has $h$ vertices. As $\mathcal{G}$ is minor closed, $\mathcal{G}$ excludes $K_{h}$ as a minor. Therefore if $G \in \sqrt{\mathcal{G}}$, then $G^{2}$ does not contain $K_{h}$ as a minor. From Lemma 1, $G$ does not contain as a subgraph a properly subdivided wall of height $\geq h-2$. From Lemma 1, bw $(G)<f(h-2)$. Notice also that $\Delta(G) \leq h-1$. Then, from Lemma 4, $\mathbf{c w}(G)<(h-1) \cdot f(h-2)$ and the Theorem follows if we set $c_{\mathcal{G}}=(h-1) \cdot f(h-2)$.

In the rest of this section we will also bound the branch-width of the squares of the graphs in $\mathcal{G}$. This will be useful for the algorithmic consequences of our results in Section 4.

Lemma 5. For every graph $G$ it holds that $\mathbf{c w}\left(G^{2}\right) \leq 2 \cdot(\mathbf{c w}(G))^{2}-\mathbf{c w}(G)$.

Proof. Let $(T, \rho)$ be a carving decomposition of $G$ of width at most $k$. We will prove that $(T, \rho)$ is a carving decomposition of $G^{2}$ of width at most $2 k^{2}-k$. We use the notation $\beta$ and $\beta^{\prime}$ in order to distinguish the bridge sets for $G$ and $G^{2}$ respectively. Let $f \in E(T)$. As $\Delta(G) \leq \mathbf{c w}(G)$, it is enough to prove that $\beta^{\prime}(f) \leq 2 \cdot k \cdot(\Delta(G)-1)+k$. To prove this, we first define $S=\rho^{-1}\left(T^{\prime}\right)$ where $T^{\prime}$ is one of the connected components of $T-\{e\}$. We define $L=S \cap\left(\bigcup_{e \in \beta(f)} e\right)$ and $R=\bar{S} \cap\left(\bigcup_{e \in \beta(f)} e\right)$. Finally, for each $e=\{x, y\} \in \beta(f)$ where $x \in L$ and $y \in R$, we define the sets

$$
\begin{aligned}
& Q_{R}(e)=\left\{\{x, z\} \mid z \in N_{G}(y) \cap \bar{S}\right\} \\
& Q_{L}(e)=\left\{\{z, y\} \mid z \in N_{G}(x) \cap S\right\}
\end{aligned}
$$


We claim that each edge $e^{\prime}=\{v, u\} \in \beta^{\prime}(f) \backslash \beta(f)$ belongs in $Q_{R}(e) \cup$ $Q_{L}(e)$ for some $e \in \beta(f)$. For this, suppose that $e^{\prime}=\{v, u\}$ where $v \in S$ and $u \in \bar{S}$. As $e^{\prime}$ is not an edge in $G$ and there is a vertex $z \in V(G)$ such that $\{v, z\},\{z, u\} \in E(G)$. If $z \in S$ then $\{z, u\} \in \beta(e)$ and therefore $e^{\prime} \in Q_{L}(e)$ for $e=\{z, u\}$. If $z \in \bar{S}$ then $\{v, z\} \in \beta(e)$ and therefore $e^{\prime} \in Q_{R}(e)$ for $e=\{v, z\}$. In both cases, the claim holds.

Notice now that for each $e=\{x, y\} \in \beta(f),\left|Q_{R}(e) \cup Q_{L}(e)\right|=\left|Q_{R}(e)\right|+$ $\left|Q_{L}(e)\right|$. As $\left|Q_{R}(e)\right| \leq\left|N_{G}(y)\right|-1 \leq \Delta(G)-1$ and $\left|Q_{L}(e)\right| \leq\left|N_{G}(x)\right|-1 \leq$ $\Delta(G)-1$, we conclude that $\left|Q_{R}(e) \cup Q_{L}(e)\right| \leq 2 \cdot(\Delta(G)-1)$. Therefore $\left|\beta^{\prime}(f) \backslash \beta(f)\right| \leq 2 \cdot k \cdot(\Delta(G)-1)$ and the claim follows.

Lemma 6. Let $\mathcal{G}$ be a class that is minor closed and let $G \in \sqrt{\mathcal{G}}$. Then bw $\left(G^{2}\right) \leq 4 \cdot c_{\mathcal{G}}^{2}-2 \cdot c_{\mathcal{G}}$.

Proof. Let $G^{2}$ be a graph in $\mathcal{G}$. From Theorem 1, $\mathbf{c w}(G) \leq c_{\mathcal{G}}$ and, from Lemma 5, $\mathbf{c w}\left(G^{2}\right) \leq 2 \cdot c_{\mathcal{G}}^{2}-c_{\mathcal{G}}$. As $G^{2}$ is a graph, we have that $\Delta^{*}\left(G^{2}\right)=2$. This together with Observation 2 and Lemma 4 implies that bw $\left(G^{2}\right) \leq 4 \cdot c_{\mathcal{G}}^{2}-2 \cdot c_{\mathcal{G}}$.

\section{Linear time recognition of $\sqrt{\mathcal{G}}$}

In this section we study the complexity of the following problem when $\mathcal{G}$ is a minor closed graph class.

SQUARE RoOT of $\mathcal{G}$
Instance: A graph $G$.
Question: is $G$ a member of $\sqrt{\mathcal{G}}$ ?

Before we deal with the above problem, we briefly expose some known facts on the recognition of minor closed graph classes. Let $\mathcal{G}$ be such a class. We denote by $\operatorname{obs}(\mathcal{G})$ the set of minor minimal graph that do not belong in $\mathcal{G}$. Notice that any two graphs in $\mathbf{o b s}(\mathcal{G})$ are incomparable with respect to the minor relation. In their Graph Minors series, Robertson and Seymour [9] proved that each such set is finite, and therefore the size of its graphs is bounded by some constant $c_{\mathcal{G}}^{\prime}$. Therefore, to check whether an input graph $G$ is a member of some minor-closed $\mathcal{G}$, it sufices to check whether some graph on $\operatorname{obs}(\mathcal{G})$ is a minor of $G$. If this is the case, return NO as answer, otherwise return YES. In the same series of papers, Robertson and Seymour proved that to check whether a fixed size graph $H$ is a minor 
of an $n$-vertex graph $G$ can be done in $O\left(n^{3}\right)$ steps. Morever, when the input graph $G$ has branchwidth at most $k$, this check can be done in time $f(k) \cdot n$, that is linear in $n$ (see e.g. [8, 1, 4]). We use this fact to prove our main algorithmic result.

Theorem 2. For every non-trivial graph class $\mathcal{G}$ that is minor closed, there exists an algorithm deciding $\sqrt{\mathcal{G}}$ in linear time.

Proof. Let $c_{\mathcal{G}}$ be the constant of Theorem 1 and let $G$ be an input graph to the problem of the recognition of $\sqrt{\mathcal{G}}$. If $\Delta(G)>c_{\mathcal{G}}$, then, from Observation 1, $\mathbf{c w}(G)>c_{\mathcal{G}}$ and the answer to the problem is NO. Assuming that $\Delta(G) \leq c_{\mathcal{G}}$, the computation of $G^{2}$ can be done in linear time. From Lemma 6. if $G \in \sqrt{\mathcal{G}}$, then the branchwidth of $G^{2}$ is at most $c_{\mathcal{G}}^{\prime}=4 \cdot c_{\mathcal{G}}^{2}-2 \cdot c_{\mathcal{G}}$. We now check whether bw $\left(G^{2}\right) \leq c_{\mathcal{G}}^{\prime}$, e.g. using the algorithm of [2]. If the answer is negative, then we safely return NO. Otherwise, it remains to check whether $G \in \mathcal{G}$ for a graph of bounded branchwidth and this can be done in linear time as commented above.

[1] Isolde Adler, Frederic Dorn, Fedor V. Fomin, Ignasi Sau, and Dimtrios M. Thilikos. Faster parameterized algorithms for minor containment. In 12th Scandinavian Workshop on Algorithm TheorySWAT 2010 (Bergen), volume 6139 of LNCS, pages 322 - 333. Springer, Berlin, 2010.

[2] Hans L. Bodlaender and Dimitrios M. Thilikos. Constructive linear time algorithms for branchwidth. In Automata, Languages and Programming, 24th International Colloquium, ICALP'97, volume 1256 of LNCS, pages 627-637. Springer, Berlin, 1997.

[3] Frank Harary, Richard M. Karp, and William T. Tutte. A criterion for planarity of the square of a graph. J. Combinatorial Theory, 2:395-405, 1967.

[4] Illya V. Hicks. Branch decompositions and minor containment. Networks, 43(1):1-9, 2004.

[5] Yaw ling Lin and Steven S. Skiena. Algorithms for square roots of graphs. SIAM Journal on Discrete Mathematics, 8:99-118, 1991.

[6] Neil Robertson and Paul D. Seymour. Graph minors. V. Excluding a planar graph. J. Comb. Theory Series B, 41:92-114, 1986. 
[7] Neil Robertson and Paul D. Seymour. Graph minors. X. Obstructions to tree-decomposition. J. Combin. Theory Ser. B, 52(2):153-190, 1991.

[8] Neil Robertson and Paul D. Seymour. Graph minors. XIII. The disjoint paths problem. Journal of Combinatorial Theory. Series B, 63(1):65110, 1995.

[9] Neil Robertson and Paul D. Seymour. Graph minors. XX. Wagner's conjecture. J. Combin. Theory Ser. B, 92(2):325-357, 2004.

[10] Paul D. Seymour and Robin Thomas. Call routing and the ratcatcher. Combinatorica, 14(2):217-241, 1994. 